\title{
ДВУХ- И ТРЕХФОТОННЫЙ ЛИНЕЙНО-ЦИРКУЛЯРНЫЙ ДИХРОИЗМ В ПОЛУПРОВОДНИКАХ КУБИЧЕСКОЙ СИММЕТРИИ *
}

\begin{abstract}
Теоретически исследован линейно-циркулярный дихроизм двух- и трехфотонного поглощения света в полупроводниках кубической симметрии дырочной проводимости. Рассчитаны матричные элементы двух- и трехфотонных оптических переходов, протекающих между подзонами валентной зоны полупроводника. При этом учтены переходы, связанные как с неодновременным поглощением отдельных фотонов, так и с одновременным поглощением двух фотонов, а также определена спектральная и температурная зависимости коэффициентов двух- и трехфотонного поглощения поляризованного излучения.
\end{abstract}

Ключевые слова: линейно- и циркулярно поляризованный свет, матричный элемент, оптический переход, вероятность перехода, носители тока, валентная зона, полупроводник.

Создание лазеров и мазеров представляют возможность проведения исследований по выстраиванию импульсов и оптической ориентации моментов носителей тока при одно- и многофотонном поглощении поляризованного излучения в полупроводниках, дающих информацию о природе электрон-фотонного взаимодействия и спин-зависимой релаксации импульса электронов [1-4].

В настоящее время многофотонный линейно-циркулярный и циркулярно-циркулярный дихроизм исследован в полупроводниках при поглощении света различной частоты и поляризации [2], обусловленный междузонными оптическими переходами, т.е. оптическими переходами между валентной зоной и зоной проводимости полупроводника. В частности, в [2] построена теория линейно-циркулярного дихроизма (ЛЦД) многофотонного межзонного поглощения света в полупроводниках в области развитой нелинейности, т.е. в области интенсивности, когда удовлетворяется условие $\frac{2 \pi e^{2} I\left|\boldsymbol{e} \boldsymbol{p}_{c v}\right|^{2}}{c n_{\omega} \omega^{2} m_{0}^{2}(\hbar \omega)^{2}}<<1$, где $\boldsymbol{e}$ и $I-$ вектор поляризации и интенсивность света; $p_{c v}=p_{c \boldsymbol{k}, v \boldsymbol{k}}=\boldsymbol{e} \boldsymbol{p}_{c \boldsymbol{k}, v \boldsymbol{k}}-$ межзонный матричный элемент оператора импульса; $n_{\omega}-$ показатель преломления света среды на частоте $\omega ; m_{0}$ - масса свободного электрона.

В вышеуказанных работах открытыми остались процессы поглощения света, обусловленные двух- и трехфотонными оптическими переходами между подзонами валентной зоны или зоны проводимости полупроводника, а также не учтено одновременное поглощение двух фотонов [511], чему посвящена настоящая работа. Далее проведем квантово-механический анализ двухи трехфотонного ЛЦД в полупроводниках со сложной валентной зоной.

Хотя однофотонное (линейное по интенсивности) поглощение поляризованного излучения в полупроводниках, обусловленное оптическими переходами между подзонами легких и тяжелых дырок валентной зоны, исследуется как теоретически, так и экспериментально уже довольно давно ([12] и ссылки в ней), вопрос о ЛЦД (см., например, [5-11]) двух- и трехфотонного поглощения света с учетом одновременного поглощения остается открытым.

Поэтому ниже рассмотрим двух- и трехфотонное поглощение поляризованного излучения в полупроводниках кубической симметрии, обусловленное прямыми оптическими переходами между подзонами легких и тяжелых дырок.

Следуя [9-11, 13-16], с учетом вклада эффекта когерентного насыщения в коэффициент $N$ фотонного поглощения света $K^{(N)}(\omega, T)$ получим

$$
K^{(N)}(\omega, T)=2 \pi N \frac{\omega}{I} \rho(N \hbar \omega) F(\beta, N, \omega) \sum_{m=\mp 1 / 2 ; m^{\prime}=\mp 3 / 2 ;}\left\langle\frac{\left|M_{m^{\prime}, m}^{(N)}(\boldsymbol{k})\right|^{2}}{\sqrt{1+4 \frac{\alpha_{\omega}}{\hbar^{2} \omega^{2}}\left|M_{m^{\prime}, m}^{(N)}(\boldsymbol{k})\right|^{2}}}\right\rangle,
$$

\footnotetext{
* Работа частично финансирована грантом ОТ-Ф2-66.
} 
где $M_{m ; m^{\prime}}^{(N)}(k)$ - составной матричный элемент оптического перехода из состояния $\left|m^{\prime} \boldsymbol{k}\right\rangle$ в $|m \boldsymbol{k}\rangle$; $F(\beta, N, \omega)=\left[1-\exp \left(N \hbar \omega /\left(k_{\mathrm{B}} T\right)\right)\right] \exp \left[\left(E_{F}-E_{1}^{(N)}\right) / k_{\mathrm{B}} T\right] ; E_{1}^{*}=m_{2} \hbar \omega\left(m_{2}-m_{1}\right) ; I=\frac{n_{\omega} \omega^{2} A_{0}^{2}}{2 \pi c}\left(A_{0}\right)-$ интенсивность (амплитуда вектора потенциала) света; $E_{l \boldsymbol{k}}-$ энергетический спектр дырок в подзоне $l\left(l=1(l=2)\right.$ - для тяжелых (легких) дырок); $n_{\omega}$ - коэффициент преломления на частоте $\omega$; $\hbar \omega$ - энергия фотона; $\rho(N \hbar \omega)=\mu_{-} k_{\omega}^{(N)} /\left(\pi^{2} \hbar^{2}\right)$ - приведенная плотность состояний фотовозбужденных дырок; $k^{(N)}=\left(2 \mu_{-} N \omega / \hbar\right)^{1 / 2} ; \quad \alpha_{\omega}=6 \omega^{2} T_{1}^{(1)} T_{2}^{(1)} \frac{I}{I_{0}}, \quad I_{0}=\frac{c n_{\omega} \hbar^{3} \omega^{3}}{2 \pi|B|}, B=\frac{\hbar^{2}\left(m_{1}-m_{2}\right)}{2 m_{1} m_{2}} ;$ знак $\langle\ldots\rangle$ означает усреднение по телесному углу волнового вектора дырок $\boldsymbol{k}$. Остальные величины общеизвестные. Здесь электронам в подзоне $l=1$ (тяжелые дырки) соответствуют состояния с проекцией $m= \pm 3 / 2$ углового момента по направлению $\boldsymbol{k}$, а электронам в подзоне $l=2$ (легкие дырки) - состояния с $m= \pm 1 / 2$. Например, для $p$-GaAs $I_{0}=13420$ кВт/см ${ }^{2}$ при $\hbar \omega=17$ мэВ, $m_{1}=0,51 m_{0}\left(m_{2}=0.09 m_{0}\right)$ - эффективная масса тяжелых (легких) дырок, $E_{1}^{(N)}=N E_{1}^{*}$.

Из (1) видно, что для определения спектральной или температурной зависимости коэффициента многофотонного поглощения света $K^{(N)}$ необходимо рассчитать матричные элементы рассматриваемых оптических переходов, которые анализируются ниже для конкретных случаев.

Следуя [11], матричный элемент двухфотонного оптического перехода представим в виде

$$
M_{m \boldsymbol{k}, m^{\prime} \boldsymbol{k}}^{(2)}=M_{m, m^{\prime}}^{(2)}=\sum_{m^{\prime \prime}= \pm 1 / 2, \pm 3 / 2} \frac{M_{m, m^{\prime \prime}}^{(1)} M_{m^{\prime \prime}, m^{\prime}}^{(1)}}{\left(E_{m^{\prime \prime} \boldsymbol{k}}-E_{m^{\prime} \boldsymbol{k}}-\hbar \omega\right)}-\left(\frac{e A_{0}}{c \hbar}\right)\left[H_{\Gamma_{6}}^{(2)}\left(\boldsymbol{e}^{\prime}\right)\right]_{m, m^{\prime}} .
$$

Здесь $H_{\Gamma_{6}}^{(2)}\left(\boldsymbol{e}^{\prime}\right)=H_{\Gamma_{6}}^{(2)}\left(\boldsymbol{k} \rightarrow \boldsymbol{e}^{\prime}\right), H_{\Gamma_{6}}^{(2)}(\boldsymbol{k})-$ эффективный гамильтониан дырок в представлении Латтинжера - Кона $[17,18] ; e_{x^{\prime}}, e_{y^{\prime}}, e_{z^{\prime}}-$ компоненты вектора $\boldsymbol{e}^{\prime}$, где $e_{x^{\prime}}, e_{y^{\prime}}-$ проекции $\boldsymbol{e}^{\prime}$ вектора поляризации света на оси $x^{\prime}, y^{\prime}$, перпендикулярные к волновому вектору дырок $(\boldsymbol{k})$. Отметим, что первое слагаемое (2) описывает двухквантовый межподзонный оптический переход, протекающий с поглощением двух одинарных фотонов, а второе слагамое - одновременное поглощение двух фотонов.

Далее проанализируем матричные элементы для различного типа двух- и трехфотонных оптических переходов в зависимости от степени поляризации света. С учетом (1) нетрудно получить выражение для усредненного по телесному углу дырок квадрата модуля матричного элемента двухфотонного оптического перехода, описываемого суммой фейнмановских диаграмм рис. $1, a, \sigma$, в виде $\frac{1}{20} \xi^{4} \mathfrak{R}$, где $\mathfrak{R}=8$ - для линейной, $\mathfrak{R}=7$ - для циркулярной поляризации, $\xi=\frac{e A_{0}}{c \hbar} \sqrt{B}$. Откуда коэффициент ЛЦД для вышеуказанных оптических переходов равен 8/7.
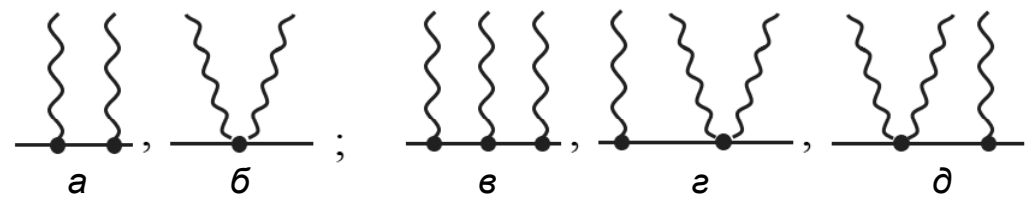

Рис. 1. Диаграммы Фейнмана, описывающие двух- $(a, \sigma)$ и трехфотонные $(b, 2, d)$ оптические переходы, происходящие между подзонами тяжелых и легких дырок валентной зоны полупроводника, где прямая линия - дырка, волнистая - фотон, диаграмма поглощение, диаграмма \{\}$-$ одновременное поглощение двух фотонов

С учетом эффекта когерентного насыщения [9-12] его вклад в матричный элемент вышеуказанных оптических переходов определяется как 


$$
\sum_{m^{\prime}= \pm 1 / 2, m= \pm 3 / 2} \delta\left|M_{m^{\prime} m}^{(N)}(\boldsymbol{k})\right|^{2}=\sum_{m^{\prime}= \pm 1 / 2, m= \pm 3 / 2} \frac{\left|M_{m^{\prime} m}^{(N)}(\boldsymbol{k})\right|^{2}}{\sqrt{1+4 \frac{\alpha_{\omega}}{\hbar^{2} \omega^{2}}\left|M_{m^{\prime} m}^{(N)}(\boldsymbol{k})\right|^{2}}}-\left|M_{m^{\prime} m}^{(N)}(\boldsymbol{k})\right|^{2} .
$$

Отметим, что для определения вероятностей оптических переходов или коэффициента поглощения света требуется провести угловое усреднение выражения (3) по телесному углу волнового вектора дырок. Эти угловые усреднения для $N=2,3, \ldots$ с учетом эффекта когерентного насыщения аналитически не имеют решения. Поэтому далее рассмотрим экспериментально интересуемую область интенсивности света, т.е. когда выполняется условие $1>>4 \frac{\alpha_{\omega}}{\hbar^{2} \omega^{2}}\left|M_{m^{\prime} m}^{(N)}(\boldsymbol{k})\right|^{2}$. Для этого удобно произвести интегрирование по телесному углу волнового вектора дырок, разлагая радикал (3) в ряд. В частности, для однофотонных оптических переходов имеем

$$
\sum_{m^{\prime}= \pm 1 / 2, m= \pm 3 / 2} \delta\left|M_{m^{\prime} m}^{(N=1)}(\boldsymbol{k})\right|^{2}=-\frac{9}{4} \frac{\alpha_{\omega}}{\hbar^{2} \omega^{2}} \xi^{8}\left[1296\left|e_{ \pm}^{\prime} e_{z^{\prime}}\right|^{4}+\left(36 e_{z^{\prime}}{ }^{2}\left|e_{+}^{\prime}\right|^{2}+\left|e_{-}^{\prime 2}\right|^{2}\right)^{2}\right]
$$

и, проведя угловое интегрирование, получим следующие соотношения:

$$
\left\langle\sum_{m^{\prime}= \pm 1 / 2, m= \pm 3 / 2} \delta\left|M_{m^{\prime} m}^{(N=1)}(\boldsymbol{k})\right|^{2}\right\rangle=-\frac{9}{4} \frac{\alpha_{\omega}}{\hbar^{2} \omega^{2}} \xi^{8} \frac{1}{315}\left\{\begin{array}{l}
29792 \text { - для линейной поляризации, } \\
30395 \text { - для циркулярной поляризации. }
\end{array}\right.
$$

При этом видно, что вклад эффекта когерентного насыщения в коэффициент двухфотонного ЛЦД в $p$-GaAs равен 0.98 .

Теперь проанализируем трехфотонные оптические переходы между подзонами тяжелых и легких дырок. Рассмотрим оптические переходы на рис. 1,6 2, $\partial$. После проведения углового усреднения по телесному углу волнового вектора дырок квадрата модуля трехфотонных оптических переходов имеем: $\frac{297}{4} \frac{\xi^{6}}{\hbar \omega}$ - для линейной; $\frac{405}{16} \frac{\xi^{6}}{\hbar \omega}-$ для циркулярной поляризации. Из последних соотношений видно, что коэффициент трехфотонного ЛЦД для этого типа оптического перехода равен 2.9.

Далее определим спектральную и температурную зависимости коэффициента двухфотонного поглощения. Следуя [9-12], определим коэффициент $N$ фотонного поглощения поляризованного света в виде

$$
K^{(N)}=\frac{2 \pi}{\hbar} \frac{\hbar \omega}{I} \sum_{\boldsymbol{k}, m= \pm 1 / 2 ; m^{\prime}= \pm 3 / 2}\left(f_{1 \boldsymbol{k}}^{(N)}-f_{2 \boldsymbol{k}}^{(N)}\right)\left|M_{m ; m^{\prime}}^{(N)}(k)\right|^{2} \delta\left(E_{2 \boldsymbol{k}}-E_{1 \boldsymbol{k}}-N \hbar \omega\right),
$$

где $f_{l \boldsymbol{k}}^{(N)}$ - неравновесная функция распределения дырок, участвующих в $N$-фотонном оптическом переходе. Тогда коэффициенты двух- и трехфотонного поглощения света без учета эффекта когрентного насыщения определяются выражениями

$$
\begin{aligned}
& K^{(N=2)}=2 \sqrt{2} \Xi_{m^{\prime} m}^{(2,1)} \exp \left[-m_{h h} m_{l h} \hbar \omega /\left(m_{h h}-m_{l h}\right) k_{\mathrm{B}} T\right] K^{(N=1)}, \\
& K^{(N=3)}=3 \sqrt{3} \Xi_{m^{\prime} m}^{(3,1)} \exp \left[-2 m_{h h} m_{l h} \hbar \omega /\left(m_{h h}-m_{l h}\right) k_{\mathrm{B}} T\right] K^{(N=1)} .
\end{aligned}
$$

Здесь $K^{(N=1)}-$ коэффициент однофотонного поглощения света $[12,13] ; \quad E_{1}^{(N=2)}=2 E_{1}^{*}$, $E_{1}^{*}=\frac{m_{h h} m_{l h}}{m_{h h}-m_{l h}} \hbar \omega ; \Xi_{m^{\prime} m}^{(2,1)}=\xi^{2}$ и $\Xi_{m^{\prime} m}^{(3,1)}=111 \cdot \xi^{4}-$ для линейной, $\Xi_{m^{\prime} m}^{(2,1)}=0.65 \xi^{2}$ и $\Xi_{m^{\prime} m}^{(3,1)}=102 \cdot \xi^{4}-$ для циркулярной поляризации. В результате имеем, что коэффициент двухфотонного ЛЦД равен 1.52 , а для трехфотонного - 1.1 .

На рис. 2 приведены спектральные зависимости $K^{(N=1)}(a), K^{(N=2)}\left(\right.$ б), $K^{(N=3)}($ (в) для $p$-GaAs при межподзонном поглощении линейно-поляризованного света для двух температур: $T=200 \mathrm{~K}$ (сплошная кривая) и $T=300 \mathrm{~K}$ (ромбики). На рис. 3 показаны температурные зависимости $K^{(N=1)}(a), \quad K^{(N=2)}(б), \quad K^{(N=3)}($ в) для $p$-GaAs при межподзонном поглощении линейно-поляризованного света для двух длин волн: $\lambda=10.6$ мкм (сплошная кривая) и $\lambda=9.5$ мкм (ромбики). 

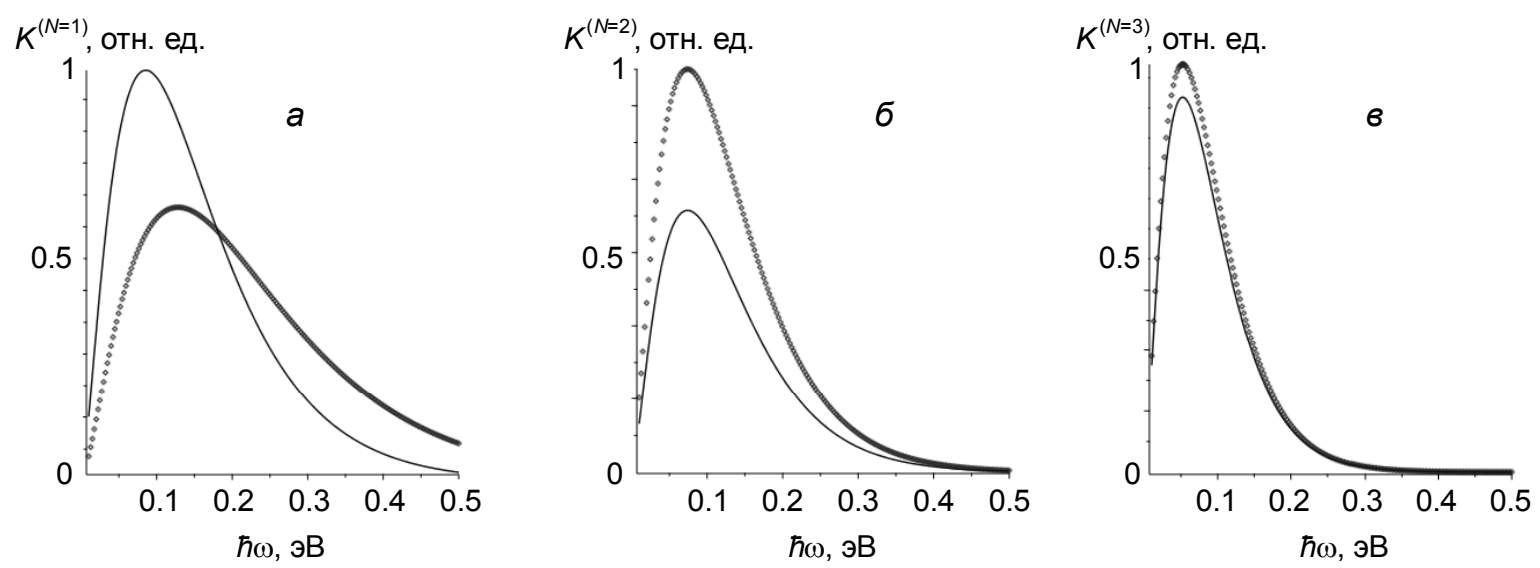

Рис. 2. Спектральные зависимости $K^{(N=1)}(a), K^{(N=2)}(\sigma), K^{(N=3)}($ в) для $p$-GaAs при межподзонном поглощении линейно-поляризованного света для двух температур: сплошная кривая - при $T=200$ К, ромбики - при $T=300 \mathrm{~K}$

Из рис. 2 и 3 видно, что спектральная (температурная) зависимость коэффициентов поглощения линейно-поляризованного света с ростом энергии фотона (температуры) сначала растет и достигает максимума, затем уменьшается. Расчеты показывают, что при снижении температуры в 1.5 раза максимальные значения в спектральных зависимостях $K^{(N=1)}(\omega), \quad K^{(N=2)}(\omega)$ умень-
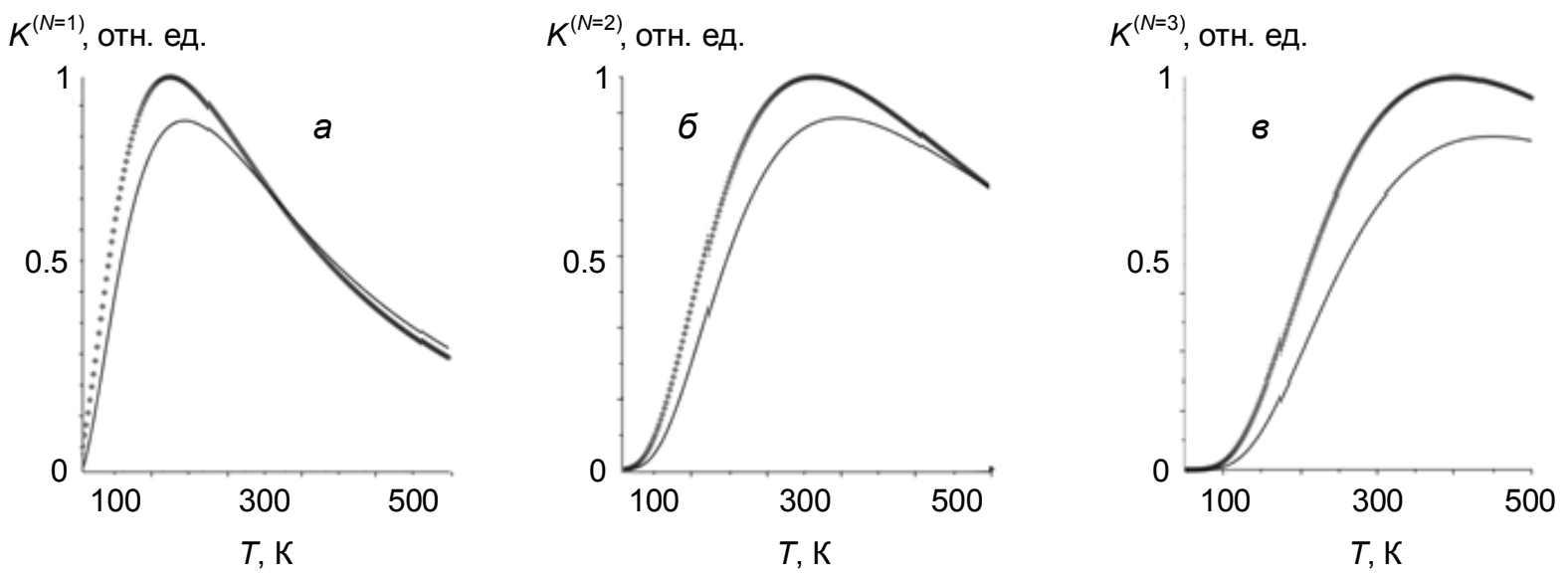

Рис. 3. Температурные зависимости $K^{(N=1)}(a), K^{(N=2)}(б), K^{(N=3)}($ в) для $p$-GaAs при межподзонном поглощении линейно-поляризованного света для двух длин волн света: сплошная кривая при $\lambda=10.6$ мкм , ромбики - при $\lambda=9.5$ мкм, $T=300 \mathrm{~K}$

шаются примерно в 1.4 раза, а для $K^{(N=3)}(\omega)$ почти не изменяются; при уменьшении длины волны света незначительно уменьшаются максимальные значения в температурных зависимостях $K^{(N=1)}(T), K^{(N=2)}(T)$ и $K^{(N=3)}(T)$. Для сопоставления $K^{(N=1)}(\omega)$ и $K^{(N=2)}(\omega)$ на рис. 4 приведены спектральные зависимости $K^{(N=1)}$ (сплошная кривая) и $K^{(N=2)}$ (ромбики) для $p$-GaAs при межподзонном поглощении линейно-поляризованного света для $T=300 \mathrm{~K}$. Видно, что при $\xi=0.1$ лишь в области малых значений частот преобладает двухфотонное поглощение над однофотонным, а далее - наоборот.

Аналогичная ситуация имеет место и для циркулярно-поляризованного света.

В заключение отметим, что пренебрежение одновременным поглощением двух фотонов может привести к заметной погрешности в расчетах коэффициента поглощения или других оптических величин, например, фототока. Таким образом: 1) вероятности многофотонных переходов зависят от степени поляризации света, т.е. имеет место ЛЦД нелинейного по интенсивности поглощения света в полупроводнике со сложной валентной зоной; 2) температурная зависимость коэффициента двух- и трехфотонного межподзонного поглощения поляризованного излучения в полупроводнике со сложной валентной зоной в области частот, когда $\hbar \omega>>k_{\mathrm{B}} T$, определяется темпе- 


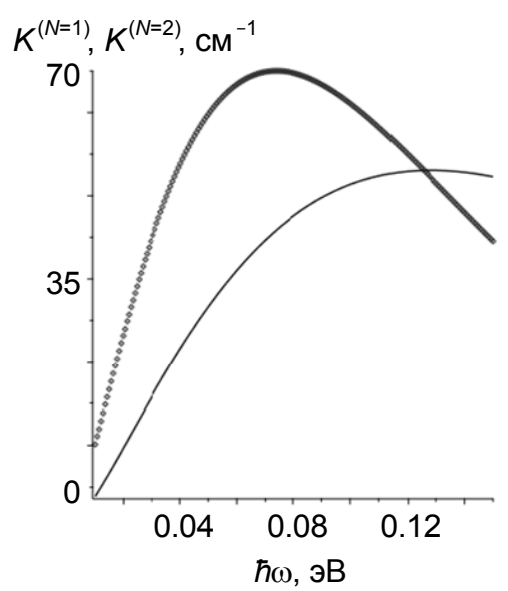

Рис. 4. Спектральные зависимости $K^{(N=1)}$ (сплошная кривая) и $K^{(N=2)}$ (ромбики) для $p$-GaAs при межподзонном поглощении линейно-поляризованного света для $T=300 \mathrm{~K}$

ратурной зависимостью коэффициента однофотонного поглощения; 3) спектральная и температурная зависимости коэффициентов поглощений линейно- и циркулярно поляризованного света с ростом энергии фотона (температуры) сначала растут и достигают максимума, а затем уменьшаются; 4) показано, что при $T=300 \mathrm{~K}$ и $\xi=0.1$ лишь в области малых значений частот преобладает двухфотонное поглощение над однофотонным (а далее, наоборот), а трехфотонное поглощение всегда меньше однофотонного.

Отметим также, что спектральная и поляризационная зависимости вероятности оптического перехода, происходящего с поглощением двух фотонов с различной частотой $\left(\omega_{1}, \omega_{2}\right)$ и поляризацией $\left(\boldsymbol{e}_{1}, \boldsymbol{e}_{2}\right)$ и обусловленного переходами валентной зоны и зоны проводимости полупроводника кубической симметрии, определяются соотношением $\left(\omega_{1}^{-2}+\omega_{2}^{-2}+\omega_{1}^{-1} \omega_{2}^{-1}\left|\boldsymbol{e}_{1} \cdot \boldsymbol{e}_{2}^{*}\right|^{2}\right)$, и в полупроводниках со сферической зонной структурой не возникает двухфотонный межзонный ЛЦД, поскольку величина $\left\langle\left|\boldsymbol{e}_{1} \cdot \boldsymbol{e}_{2}^{*}\right|^{2}\right\rangle$ не зависит от степени поляризации света и формируется при учете эффекта когерентного насыщения.

\section{СПИСОК ЛИТЕРАТУРЫ}

1. Ivchenko E. L. Optical Spectroscopy of Semiconductor Nanostructures. - Harrow : Alpha Science International Ltd., 2005. - XII. -427 p.

2. И в ч е н к о Е. Л. Новые оптические явления в полупроводниках: дис. ... д.ф.-м.н. - Л., 1983. - 148 с.; И в ч е н к о Е. Л. // ФТТ. - 1972. - Т. 14. - Вып. 12. - С. 3489.

3. S p i n Physics in Semiconductors / ed. M.I. Dyakonov. - Heydelberg: Springer Verlag, 2008. - 447 p.; O p t i c a 1 orientation / eds. F.Meier and B.P. Zakharchenya. - North-Holland; New York; Tokyo, 1984. - 534 p.

4. Шалыг и н В. А. Оптические и фотогальванические эффекты в объемных полупроводниках и двумерных структурах: автореф. дис. ... докт. физ.-мат. наук. - СПб., 2013. - 34 с.

5. Raluca A. Negres A., Joel M. Hales, Andrey Kobyakov, et a l. // IEEE J. Quantum Electron. - 2002. - V. 38. - P. 205-1209.

6. Jun He, Yingli Qu, Heping Li, et a 1. // Opt. Soc. Am. - 2005. - V. 13. - P. 9235-9241.

7. Hurlbut W.C., Yun-Shik Lee, Vodopyanov K.L., et a1. // Opt. Lett. - 2007.-V. 32. - P. 668673.

8. Shaul Pearl, Nir Rotenberg, and Henry M. van Driel // Appl. Phys. Lett. - 2008. - V. 93. P. 131102-131109.

9. Rasulov V.R., Rasulov R.Ya., and Eshboltaev I. // Phys. Solid State. - 2017. - V. 59. - No. 3. P. 463-468.

10. Р асулов Р.Я., Р асулов В.Р., Эшбол та ев И. // Изв. вузов. Физика. - 2015. - Т. 58. - № 12. C. $13-17$.

11. Р а с улов Р.Я. // ФТТ. - 1993. - Т. 35. - Вып. 6. - С. 1107.

12. Р а с у л о в Р. Я . Поляризационные оптические фотогальванические эффекты в полупроводниках при линейном и нелинейном поглощении света: дис. ... докт. физ.-мат. наук. - СПб.: ФТИ РАН им. акад. А.Ф. Иоффе, 1993. $168 \mathrm{c}$.

13. Ганичев С.Д., Ивченко Е.Л., Р асулов Р.Я. и др. //ЖЭТФ. - 1993. - Вып. 1. - С. 198. 
14. Rasulov V.R., Rasulov R.Ya., and Eshboltaev I. // Semiconductors. - 2016. - V. 50. - No. 2. P. $145-153$.

15. Р асулов Р.Я., Р асул ов В.Р., Эшбол та ев И. // Изв. вузов. Физика. - 2016. - Т. 59. - № 1. - С. $77-82$.

16. Р а сул о в В.Р., Р а сул ов Р.Я., Эшб ол та е в И. М. // Изв. вузов. Физика. - 2016. - Т. 59. - № 3. C. $114-121$.

17. Би р Г. Л ., П и ку с Г. Е . Симметрия и деформационные эффекты в полупроводниках. - М.: Наука, 1973.

18. И в че н к О Е. Л., Р а сулов Р.Я. Симметрия и реальная зонная структура полупроводников. - Ташкент: Фан, 1989. - $126 \mathrm{c}$.

${ }^{1}$ Ферганский государственный университет,

Поступила в редакцию 24.02.2020, после доработки - 15.06.2020.

г. Фергана, Республика Узбекистан

${ }^{2}$ Кокандский государственный педагогический институт,

г. Коканд, Республика Узбекистан

Расулов Рустам Явкачович, д.ф.-м.н., профессор, профессор каф. физики ФерГУ, e-mail: r_rasulov51@mail.ru;

Расулов Вохоб Рустамович, $\mathrm{PhD}$, доцент каф. физики ФерГУ, e-mail: r_rasulov51@mail.ru;

Эшболтаев Икболжон, $\mathrm{PhD}$, ст. преподаватель каф. методики преподавания физики и астрономии КГПИ, е-таil: r_rasulov51@mail.ru;

Султонов Равшан Рустамович, преподаватель каф. методики преподавания физики и астрономии КГПИ, е-таil: r_rasulov51@mail.ru. 